\title{
SOZIALUNTERNEHMEN
}

\section{Den Sozialraum gestalten}

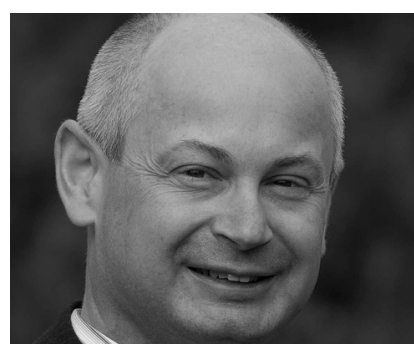

VON ULRICH KUHN

Der Diplom-Verwaltungswissenschaftler Ulrich Kuhn leitet bei der Stiftung Liebenau in Meckenbeuren (Baden-Württemberg) die Stabsstelle Sozialpolitik. Er ist zugleich Geschäftsführer von „Netzwerk: Soziales neu gestalten (SONG) «. An dem Netzwerk sind die Stiftung Liebenau, die Bremer Heimstiftung, die CBT Caritasbetriebsführungs- und Trägergesellschaft Köln, das Evang. Johanneswerk Bielefeld, die Bank für Sozialwirtschaft und das Kuratorium Deutsche Altershilfe beteiligt. Der Zusammenschluss hat sich zum Ziel gesetzt, sozialraumorientierte Strukturen zu entwickeln und innovative Modelle umzusetzen.

Internet http://www.zukunftquartier.de
Die Kommunalisierung ist eine Herausforderung für das einzelne Sozialunternehmen. Die zunehmende Differenzierung und Dezentralisierung sozialer Dienstleistungen erfordern neue Antworten insbesondere beim Rollenverständnis sozialer Träger, bei der Angebotspalette, bei der Personalentwicklung, bei der internen Organisation, bei der Finanzplanung sowie bei der Unternehmenskommunikation.

Soziale Probleme dort lösen, wo sie entstehen - auf diesen Nenner können die verstärkten Bestrebungen zur Kommunalisierung sozialer Aufgaben gebracht werden.

Der Trend ist jedoch nicht eindeutig. Aus Gründen der besseren finanziellen Absicherung spezieller Bedarfslagen oder des Ausgleichs örtlicher Ungleichheiten wurden in den vergangenen Jahrzehnten Aufgaben von den örtlichen kommunalen Gebietskörperschaften auf überregionale Ebenen verlagert (Beispiele: Einführung der Pflegeversicherung, Ansiedlung der Eingliederungshilfe bei Landeswohlfahrtsverbänden).

Insbesondere seit den Arbeitsmarktreformen 2005 verstärkt sich nun aber in verschiedenen Bereichen der Trend, soziale Aufgaben wieder auf die örtliche Ebene zu verlagern. Da die dabei verfolgten Zielsetzungen sehr differenziert sind, müssen die Erbringer sozialer Dienstleistungen zunehmend komplexeren Anforderungen genügen und ihre Strategien und Arbeitsweisen neu justieren.

\section{Näher am Menschen: sozialfachliche Erwartungen}

Nicht ohne Grund spielte die kommunale Ebene bei der Umsetzung sozialer Leistungen schon immer eine wesentliche Rolle. Die Ortsnähe ermöglicht es, bürgernah soziale Problemlagen erkennen und gezielt intervenieren zu können. Der Ausbau der großen national geregelten Säulen unseres sozialen Sicherungssystems hat zu einer hohen Versorgungssicherheit, aber auch zu Verrechtlichung, Versäulung und entsprechender Institutionenmacht geführt.

Der jüngere sozialfachliche Paradigmenwechsel hin zu Selbstbestimmung und
Teilhabe und einer an Bürgerrechten orientierten Sozialpolitik, wie sie beispielsweise in der vor einem Jahr ratifizierten UN-Behindertenrechtskonvention vorgegeben ist, rückt hingegen das örtliche Gemeinwesen zentral in den Fokus. Inklusion aller, also die möglichst umfassende Teilhabe und Teilgabe, beispielsweise auch von Menschen mit Behinderung, am Leben in der Gemeinschaft erfordert ein hierzu befähigtes und befähigendes Gemeinwesen. (1)

Sozialraumorientierte Konzepte setzen auf die lokalen sozialen Beziehungsnetzwerke und die Entwicklung von Hilfearrangements aus informellem und formellem System. Lokale Verantwortungsgemeinschaften im Sinne eines Welfare-Mix von Familie, bürgerschaftlich Engagierten und professionellen sozialen Diensten können in Wohnquartieren und Nachbarschaften Teilhabe fördern und einen sozioökonomischen Mehrwert gegenüber herkömmlichen Versorgungsmodellen erzeugen. (2)

Angesichts der Grenzen des versorgenden Sozialstaats einerseits und der familiären Solidaritätsbereitschaft andererseits wird in dieser Perspektive sozialpolitisch eine Rekonzeptionalisierung des Subsidiaritätsprinzips erforderlich, im Sinne einer Stärkung der lokalen Ebene, aber ohne Abbau sozialstaatlicher Garantiefunktionen. (3)

\section{Mehr Wirkung - Erwartungen an die kommunale Steuerung}

Vor dem Hintergrund zunehmender Finanzierungsprobleme in den Sozialhaushalten erhoffen sich die öffentlichen Kostenträger neben den genannten inhaltlichen Vorteilen Effizienzsteigerungen im Hilfesystem. Durch eine Kommunalisierung sollen Ressourcen vor Ort gebündelt, bürgerschaftliches Engagement aktiviert und eingebunden sowie eine wirksame Steuerung des Hilfesystems erreicht werden.

Neue Steuerungsmodelle im Sinne von »Local Governance « zielen darauf ab, die sozialen Dienstleistungsunternehmen in örtliche Kooperationsstrukturen einzubinden und ihnen Ergebnisverantwortung zu übertragen. (4) 
Dies soll nicht zuletzt der Begrenzung öffentlicher Sozialbudgets dienen. So waren beispielsweise mit der im Jahr 2005 erfolgten Kommunalisierung der Eingliederungshilfe in Baden-Württemberg neben einem bedarfsgerechteren Ausbau der lokalen Behindertenhilfeangebote eine effektivere Vernetzung vor Ort und Kostenbegrenzungen durch die Integration der Zuständigkeit von Leistungssteuerung und Kostenträgerschaft beim örtlichen Sozialhilfeträger intendiert. Auch die Eckpunkte der BundLänder-Arbeitsgruppe der Arbeits- und Sozialministerkonferenz zur Weiterentwicklung der Eingliederungshilfe zielen inhaltlich auf einen inklusiven Sozialraum durch den Auf- und Ausbau sozialräumlicher Unterstützungsformen, »HilfemixStrukturen « und differenzierter gemeindenaher Wohnformen ab. Gleichzeitig wird die Kooperation der Leistungsträger und Leistungsanbieter als unabdingbar bezeichnet und die Gesamtsteuerungsverantwortung und die Wirkungskontrolle durch die Träger der Sozialhilfe betont (wobei offenbleibt, ob der örtliche oder überörtliche Träger künftig zuständig sein soll). (5)

\section{Was Sozialunternehmen künftig leisten müssen}

Die Prozesse der Kommunalisierung sozialer Aufgaben verlaufen in den verschiedenen Sektoren und Regionen nicht einheitlich. Je nachdem welche Akteure die Entwicklung vorantreiben, divergieren Ausmaß, Art und Ziele und damit auch die Anforderungen an die Sozialunternehmen. Es soll im Nachfolgenden dennoch versucht werden, einige wesentliche Herausforderungen und mögliche Reaktionen von Sozialunternehmen auf die Entwicklung zu skizzieren. Dabei besteht noch ein erheblicher Entwicklungs- und Erprobungsbedarf, um geeignete Prozesse und Strukturen zu finden.

- Aufgabe und Rolle: Eine Kommunalisierung mit den genannten Zielsetzungen erfordert die strategische Weiterentwicklung des Aufgaben- und Rollenprofils sozialer Unternehmen. Innovative Träger verändern ihre Rolle - weg vom bloßen Dienstleister hin zum Partner in der Bürgergesellschaft. Sie müssen einerseits ihre bisherigen sektorspezifischen Versorgungsformen transformieren $\mathrm{zu}$ sozialräumlich ausgerichteten, personenzentrierten Dienstleistungsangeboten. Und andererseits werden sie zunehmend zum zivilgesellschaftlichen Akteur. Im Sinne einer »Enabling Agen$c y$ « (6) haben gerade die gemeinnützigen Träger die Aufgabe, inklusive Prozesse und neue Solidaritätsstrukturen in den örtlichen Gemeinwesen zu fördern. So- ziale Dienstleistungsunternehmen entwickeln sich somit zu intermediären $\mathrm{Or}$ ganisationen, die den Funktionslogiken von Staat, Markt und Zivilgesellschaft gleichzeitig gerecht werden müssen.

- Angebote: Insbesondere große Komplexeinrichtungen, die bisher umfassende Versorgungsangebote zentral für einen überregionalen Einzugsbereich vorgehalten haben, müssen ihre Wohn- und Assistenzangebote differenzieren und dezentralisieren. Die Kommunalisierung der Eingliederungshilfe in Baden-Württemberg hat beispielsweise dazu geführt, dass die nun zuständigen Stadt- und Landkreise zunehmend ihre Strukturverantwortung wahrnehmen, Behindertenhilfeplanungen umsetzen und Wert darauf legen, dass die von ihnen finanzierten Angebote für ihre Bürger auch im jeweiligen Landkreis vorhanden sind. Das bedeutet Rückbau oder gar Konversion von Komplexeinrichtungen und Ausbau der Angebote in den jeweiligen Herkunftskommunen der Leistungsempfänger. An die Stelle standardisierter Voll- leistungen in den kleinen Lebenskreisen also künftig in geteilter Verantwortung gemeinsam mit den verschiedenen Akteuren aus den informellen sozialen Netzwerken der assistenzbedürftigen Menschen. Diese Netzwerke gilt es zu knüpfen und zu moderieren. Für die Träger (und die sozialen Bildungsinstitutionen) bedeutet dies, geeignete Aus-, Fortund Weiterbildungsangebote zu entwickeln. Eine weitere Herausforderung wird es sein, für die in den gemischten Hilfearrangements tätigen Personengruppen wie Angehörige, Nachbarn, bürgerschaftlich Engagierte und bezahlt Tätige unterschiedlicher Qualifikation die Rollen und Aufgaben transparent zu differenzieren und adäquate monetäre und nicht-monetäre Honorierungen zu gestalten.

- Organisation: Um die beschriebenen Angebotsveränderungen realisieren zu können, sind entsprechende Organisationsveränderungen insbesondere in den größeren Sozialunternehmen erforderlich. Die Strukturen müssen kleinteiliger

\section{"Sozialunternehmen müssen weg von ihrer Rolle des bloßen Dienstleister hin zum Partner in der Bürgergesellschaft"}

versorgungsangebote tritt dabei eine differenzierte Palette von flexiblen Dienstleistungsmodulen, die personenorientiert von niederschwelligen, ambulanten Hilfen über teilstationäre Angebote bis zu kleinstrukturierten stationären Formen reichen kann. Im Sinne der Einbindung des Gemeinwesens wirken die Träger darüber hinaus an der Entwicklung des jeweiligen Sozialraums mit, übernehmen Funktionen in der Quartiersarbeit, fördern bürgerschaftliches Engagement und koordinieren Hilfenetzwerke aus Bürgern und Profis.

- Mitarbeiterinnen und Mitarbeiter: Die hauptberuflich Beschäftigten werden beim Aufbau von Strukturen im Gemeinwesen mitwirken, die assistenzbedürftigen Menschen ein weitestgehend gleichberechtigtes Leben in Gemeinschaft ermöglichen. Das heißt: So viel Hilfemix wie möglich und so viel professionelle Hilfe wie nötig. Die Rolle verändert sich dahingehend, dass die Profis nicht nur qualifizierte Pflege und Betreuung zu leisten haben, sondern mehr und mehr zum Moderator und Manager des Systems werden. Die im Sozialraum tätigen Assistenten erbringen ihre Assistenz- werden, indem sie sich weiter ausdifferenzieren und lokale, überschaubare, gemeindenahe Einheiten bilden. Es wird beispielsweise durch Modellprojekte - zu überprüfen sein, inwieweit die bisherige auf die sozialfachliche Spezialisierung ausgerichtete Spartenorganisation (Alten-, Behinderten-, Jugendhilfebereich etc.) durch Regionalstrukturen abgelöst oder ergänzt werden sollte. Sozialraumorientierte Arbeit richtet den Fokus nicht nur auf die speziellen Bedarfe des Einzelfalls, sondern auf die Ressourcen des Sozialraums, von denen alle Personengruppen profitieren können. Insofern wären durch die fachübergreifende Bündelung der Aktivitäten vor Ort Synergieeffekte und Betriebsgrößen denkbar, die das kleinteilige Handeln wiederum ökonomisch gestaltbar werden lassen. Die künftige Doppelrolle als Dienstleister und Inklusionsagentur erfordert innerorganisatorisch aufgrund der unterschiedlichen Handlungslogiken allerdings entsprechende Arbeitsteilungen, wodurch die Entwicklung hybrider Organisationsstrukturen befördert wird. (7) Darüber hinaus wird die Bereitschaft zur (sektorenübergreifenden) Kooperation mit un- 
terschiedlichen lokalen Akteuren und die Entwicklung geeigneter Arbeitsformen unabdingbar, wenn lokale Netzwerke gebildet und das Gemeinwesen mitgestaltet werden sollen. (8)

- Finanzierung: Im Unterschied zu standardisierten Vollversorgungssystemen führen die personenzentrierten, modulhaften Leistungsangebote sowohl zu höheren Erlösschwankungen als auch zu höherer Transparenz und einem größeren Rechtfertigungsbedarf für die Leistungsentgelte. Gleichzeitig vergrößert sich durch die Entscheidungsverlagerung auf die kommunale Ebene die Zahl der Vereinbarungspartner und entsprechend die Häufigkeit von Entgeltverhandlungen. Ein professionelles Controlling zur finanziellen Steuerung wird so ebenso unabdingbar wie die Kompetenz zur professionellen Entgeltkalkulation und Entgeltverhandlung. Für das sozialräumliche Arbeiten, insbesondere für Gemeinwesenarbeit und Netzwerkkoordination gibt es bislang keine ausreichende Refinanzierung. Sozialraumbudgets können ein Ansatz sein, wenn damit nicht sämtliche Finanzierungsrisiken von der Kommune auf die Träger verlagert werden. Für die beschriebene zivilgesellschaftliche Moderations- und Agenturfunktion sind neben kommunaler Finanzierung auch Mischfinanzierungen unter Beteiligung der Akteure der Zivilgesellschaft (Stiftungen, Unternehmen, Spenden aus der Bürgerschaft) denkbar. (9)
- Kommunikation: Mit der Kommunalisierung verändert sich die StakeholderLandschaft der Sozialunternehmen deutlich. Entsprechend ist die Kommunikation und Lobbyarbeit verstärkt auf die lokale Ebene zu fokussieren. Die Führungskräfte auf der Regional- und Ortsebene müssen mit entsprechender Vertretungskompetenz ausgestattet sein und die lokalen Netzwerkkontakte pflegen und Öffentlichkeitsarbeit betreiben. Bei Trägern, die auf unterschiedlichen sozialen Feldern tätig sind, kommt es wesentlich darauf an, dass sich die jeweils Zuständigen in der Kommunikation und Lobbyarbeit vor Ort abstimmen. Auch die Zusammenarbeit zwischen den verschiedenen vor Ort vertretenen Trägern und Verbänden der Freien Wohlfahrtspflege bei der Mitwirkung in Prozessen der kommunalen Sozialplanung ist für eine erfolgreiche Interessenvertretung unabdingbar.

Auf der Einzelfallebene - so hat die Kommunalisierung der Eingliederungshilfe in Baden-Württemberg gezeigt - wird verstärkt das Instrument des Fallmanagements und der individuellen Hilfeplanung eingesetzt. Wenn sich die Träger in den jeweiligen Hilfeplanverfahren mit flexiblen, individuell gestaltbaren Assistenzangeboten einbringen, können sie passgenaue Hilfen ermöglichen und so ihre Marktposition sichern. Zur Stärkung von Selbstbestimmung und Teilhabe müssen dabei für die Planung und Gestaltung der Angebote vor allem auch geeignete Möglichkeiten zur

\section{Bundesebene}

Sozialleistungsrechtliche Voraussetzungen, Finanzierungskonzept, Generierung von Wissenbeständen und ihre Verfügbarkeit

4 Landesebene

Ordnungsrechtliche Vorgaben, Kompetenzstrukturen, Planungsverpflichtungen,

Förderinstrumente

3

Kommunale Ebene

Planung, Transparenz, Gouvernance

2 Quartiersebene

Investitionen in die soziale Architektur

Vernetzung und Kooperation

Kulturentwicklung

1 Fallebene

Eigenverantwortung des Einzelnen und seiner Familie und Netzwerke

Soziale Sicherung für Care Leistungen im Zuzahlungsmodus

Gratifikationsleistungen auf Kindergeldniveau

Sachleistungen nur für Fachleistungen i.e.S. mit Budgetoption

Die teilhabeorientierte Gestaltung sozialer Hilfen im Welfare-Mix braucht eine neue Architektur sozialpolitischer und sozialrechtlicher Steuerung, die die gleichwertigen, aber unterschiedlichen Ebenen berücksichtigt.

Quelle: Netzwerk: Soziales neu gestalten (Hg.) (2009): Zukunft Quartier - Lebensräume zum Älterwerden, Expertise von Prof. Dr. Thomas Klie: Sozialpolitische Neuorientierung und Neuakzentuierung rechtlicher Steuerung. Gütersloh.

Partizipation der Betroffenen entwickelt werden.

\section{Kein Sozialabbau durch Kommunalisierung - Notwendigkeit übergeordneter Lobbyarbeit}

Die Chancen der Kommunalisierung sozialer Aufgaben liegen einerseits in der Stärkung von Teilhabemöglichkeiten im Gemeinwesen und der Gestaltung passgenauer Hilfearrangements. Andererseits kann der Prozess als Instrument zum Abbau gesicherter sozialer Standards genutzt werden. Es besteht die Gefahr, dass die in den vergangenen Jahren finanziell und strukturell geschwächten Kommunen nur eingeschränkt in der Lage sind, die sozialstaatlichen Defizite auf Bundes- oder Landesebene auszugleichen. (10)

Die sehr unterschiedliche Finanzkraft der Kommunen kann dazu beitragen, dass das Ziel gleicher Lebensverhältnisse in Deutschland immer weniger erreicht wird und die Zivilgesellschaft teilweise als Lückenbüßer für wegbrechende soziale Leistungen dienen soll. In Baden-Württemberg fehlt beispielsweise nach der Kommunalisierung der Eingliederungshilfe ein geeignetes Verfahren für die abgestimmte Planung für nach wie vor erforderliche überregionale Spezialversorgungsangebote für Menschen mit hohem und komplexem Hilfebedarf.

Daher darf trotz der zunehmend notwendigen kommunalen Interessenvertretung die überregionale sozialpolitische Lobbyarbeit nicht vernachlässigt werden. Durch Monitoring, Sozialberichterstattung und die Evaluation der neuen kommunalen Versorgungsstrukturen müssen die Wirkungen der Transformationsprozesse analysiert werden. Auf dieser Basis kann und muss auf Landes- und Bundesebene auf geeignete Rahmenvereinbarungen und eine insgesamt stimmige soziale Ordnungspolitik durch die Freie Wohlfahrtspflege hingewirkt werden. (11)

\section{Anmerkungen}

(1) Vgl. Evangelische Stiftung Alsterdorf/Katholische Hochschule für Sozialwesen Berlin (2009): Enabling Community - Gemeinwesen zur Inklusion befähigen! Berlin/Hamburg.

(2) Vgl. Netzwerk: Soziales neu gestalten (Hg.) (2009): Zukunft Quartier - Lebensräume zum Älterwerden, Band 3: Soziale Wirkung und »Social Return «. Gütersloh.

(3) Vgl. Netzwerk: Soziales neu gestalten (Hg.) (2009): Zukunft Quartier - Lebensräume zum Älterwerden, Experti- 
se von Prof. Dr. Thomas Klie: Sozialpolitische Neuorientierung und Neuakzentuierung rechtlicher Steuerung. Gütersloh.

(4) Dahme, H.-J., Schütter, S., Wohlfahrt N. (2008): Lehrbuch Kommunale Sozialverwaltung und Soziale Dienste. Kapitel 13: Local Governance: Dezentralisierung als Instrument einer neuen Sozialpolitik und Folgen für die Sozialen Dienste, S. 179-189. Weinheim/München.

(5) Vgl. Beschlussprotokoll der 86. Konferenz der Ministerinnen und Minister, Senatorinnen und Senatoren für Arbeit und Soziales der Länder am 25./26. November 2009 in Berchtesgaden

(6) Vgl. Evangelische Stiftung Alsterdorf/Katholische Hochschule für Sozialwesen Berlin (2009) a. a. O.

(7) Vgl. Klie, Th., Roß, P.-S. (2007): WelfareMix: Sozialpolitische Neuorientierung zwischen Beschwörung und Strategie, in: Klie, Th., Roß, P.-S. (Hg.): Sozialarbeitswissenschaft und angewandte Forschung in der sozialen Arbeit, S. 67-108. Freiburg im Breisgau.

(8) Vgl. Netzwerk: Soziales neu gestalten (Hg.) (2008): Zukunft Quartier - Lebensräume zum Älterwerden, Themenheft 2: Gemeinsam mehr erreichen - Lokale Vernetzung und Kooperation. Gütersloh.

(9) Vgl. Kuhn, U. (2007): Alternative Konzeption und Finanzierung generationsübergreifender Wohn- und Quartierskonzepte, in: Sozialwirtschaft Zeitschrift für Sozialmanagement 2/2007. Baden-Baden.

(10) Vgl. Dahme H.-J., Wohlfahrt N. (2010): Dezentralisierung und Kommunalisierung: Die Aktivierung des Lokalen als Aufgabe und strategische Ressource bürgerschaftlicher Sozialpolitik, in: Dahme H.-J., Wohlfahrt N. (Hg.): Regiert das Lokale das Soziale?, S. 26-41. Baltmannsweiler.

(11) Vgl. hierzu auch Manderscheid, H. (2010): Kommunalisierung als Implementierungsprozess - Folgen und Herausforderungen für die Freie Wohlfahrtspflege, in: Dahme H.-J., Wohlfahrt N. (Hg.): Regiert das Lokale das Soziale?, S. 42-52. Baltmannsweiler.

\section{Edition Sozialwirtschaft}

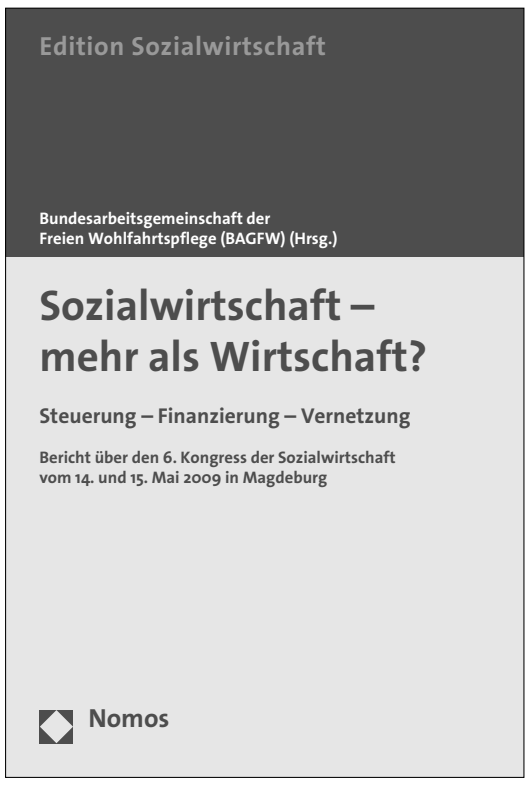

\author{
Sozialwirtschaft - \\ mehr als Wirtschaft? \\ Steuerung - Finanzierung - \\ Vernetzung \\ Bericht über den 6. Kongress der \\ Sozialwirtschaft vom 14. und 15. \\ Mai 2009 in Magdeburg \\ Herausgegeben von der \\ Bundesarbeitsgemeinschaft \\ der Freien Wohlfahrtspflege \\ (BAGFW) \\ 2010, Band 27, 283 S., brosch., \\ 49,- $€$, ISBN 978-3-8329-5257-O
}

Die Gesellschaft ist lebensnotwendig auf Grundlagen wie Vertrauen und Solidarität angewiesen, die Staat und Markt allein nicht schaffen und erhalten können. Es ist Aufgabe der Sozialwirtschaft, bei der Produktion dieses gesellschaftlichen Mehrwerts mitzuwirken. Das ist ein Ergebnis des 6. Kongresses der Sozialwirtschaft, der im Jahre 2009 in Magdeburg stattfand. Soziale Dienste und Einrichtungen müssen zwar, wie jedes Unternehmen, wirtschaftlich arbeiten, aber ihre Daseinsberechtigung erhalten sie erst dadurch, dass sie Menschen in prekären Lebenslagen helfen, ihre Notsituationen zu überwinden.

Der 6. Kongress der Sozialwirtschaft beschäftigte sich deshalb unter dem Haupttitel „Sozialwirtschaft - mehr als Wirtschaft?“ mit der Frage nach dem „Alleinstellungsmerkmal“ sozialer Träger. Nachgegangen wurde der Frage, ob sich durch die Ökonomisierung in den letzten Jahren die Unterschiede zwischen gewerblichen, kommunalen und gemeinnützigen Anbietern abgeschliffen haben. Der verstärkte Wettbewerb habe jedoch, so die Ansicht von Referenten und Kongressteilnehmern, eher zu einer Besinnung auf die je spezifischen Potenziale geführt.

Die Sozialwirtschaft zählt zu den wichtigsten Wirtschaftszweigen in der Bundesrepublik Deutschland. Der Anteil der Anbieter von Sozial- und Gesundheitsleistungen an der Bruttowertschöpfung beträgt circa 152 Milliarden Euro. Das sind rund sieben Prozent der gesamten Bruttowertschöpfung.

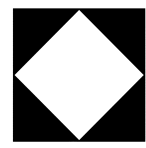

Nomos

Bitte bestellen Sie im Buchhandel oder

versandkostenfrei unter $\checkmark$ www.nomos-shop.de 


\section{Baden-Württemberg: Gemischte Bilanz}

Bereits im Jahre 2005 wurde in Baden-Württemberg die Eingliederungshilfe nach SGB XII regionalisiert. Mit dem Gesetz zur Auflösung der Landeswohlfahrtsverbände veränderten sich mit einem Schlag die Einfluss- und Finanzierungsstrukturen gründlich. Nach Jahrzehnten der Zusammenarbeit zwischen den beiden Kommunalverbänden, dem Landeswohlfahrtsverband Baden (Karlsruhe) und dem Landeswohlfahrtsverband Württemberg-Hohenzollern
(Stuttgart), und den vielen kleinen und großen Trägern der Behindertenhilfe hatte der Gesetzgeber das Spiel neu geordnet. Mit einem Federstrich wuchs die Zahl der Verhandlungs- und Geschäftspartner von zwei auf bis zu 44 Stadt- und Landkreise. Martin Beck erfragte ein Jahr später für den Informationsdienst SOZIALwirtschaft aktuell (18/2006) stichwortartig die Erfahrungen der (neuen) Leistungsträger und der (alten) Leistungsanbieter.

\section{Kommunalisierung der Eingliederungshilfe in Baden-Württemberg: was die Leistungsanbieter sagen}

\begin{tabular}{|c|c|c|c|c|c|c|c|}
\hline & $\begin{array}{l}\text { Otto Weihing, } \\
\text { Geschäftsfiührer, } \\
\text { Lebenshilfe Tuttlin- } \\
\text { gen e. V. }\end{array}$ & $\begin{array}{l}\text { Albert Ebinger, Vorstand } \\
\text { Behindertenzentrum } \\
\text { Stuttgart e. V. }\end{array}$ & $\begin{array}{l}\text { Ursula Belli, Geschäfts- } \\
\text { führerin, } \\
\text { Zieglersche Anstalten } \\
\text { Behindertenhilfe gGmbH }\end{array}$ & $\begin{array}{l}\text { Georg Schulte-Kemna, Brud- } \\
\text { erhausDiakonie, Reutlingen }\end{array}$ & $\begin{array}{l}\text { Martin Henke, Vorstand, } \\
\text { Mariaberger Heime e.V. }\end{array}$ & $\begin{array}{l}\text { Norbert Rapp, Vorstand, } \\
\text { Stiftung St. Franziskus, } \\
\text { Heiligenbronn }\end{array}$ & $\begin{array}{l}\text { Dr. Ulrich Raichle, } \\
\text { Vorstandsmitglied, } \\
\text { Diakonie Stetten e. v. }\end{array}$ \\
\hline $\begin{array}{l}\text { Wie hat sich die } \\
\text { Zahl Ihrer öffentli- } \\
\text { chen Partner (von } \\
\text { bisher zwei } \\
\text { Landeswohlfahr- } \\
\text { tsverbänden) } \\
\text { verändert? }\end{array}$ & $\begin{array}{l}\text { Wir arbeiten mit vier } \\
\text { Landkreisen zusam- } \\
\text { men. }\end{array}$ & $\begin{array}{l}\text { Wir haben es jetzt mit } 21 \\
\text { verschiedenen Kosten- } \\
\text { trägern zu tun. }\end{array}$ & $\begin{array}{l}\text { Wir haben es in Baden-Würt- } \\
\text { temberg mit } 46 \text { ver- } \\
\text { schiedenen Kostenträgern zu } \\
\text { tun. Unsere } 406 \text { Bewohner } \\
\text { sind auf diese } 46 \text { Kosten- } \\
\text { träger verteilt. }\end{array}$ & \begin{tabular}{|l} 
Die BruderhausDiakonie ist \\
derzeit in 10 Landkreisen mit \\
Angeboten der Eingliede- \\
rungshilfe tätig und hat sich \\
darüber hinaus mit dem \\
jeweiligen Landratsant bzw. \\
der Stadt zu verständigen.
\end{tabular} & \begin{tabular}{|l|} 
Bisher hatte Mariaberg in \\
Baden-Württemberg \\
vornehmlich die beiden LWV \\
als Kostenträger. Jetzt haben \\
wir es mit 37 örtlichen \\
Trägern der Eingliederungs- \\
hilfe zu tun. \\
\end{tabular} & $\begin{array}{l}\text { Seit Auflösung der Landes- } \\
\text { wohlfahrtsverbände erhöhte } \\
\text { sich die Zahl der Ansprech- } \\
\text { partner für die Stiftung auf } \\
\text { insgesamt } 38 \text { Landkreise. }\end{array}$ & $\begin{array}{l}\text { Wir haben es mit } 39 \text { der } 44 \\
\text { Stadt- und Landkreise des } \\
\text { Landes zu tun. }\end{array}$ \\
\hline $\begin{array}{l}\text { Was bedeutet das } \\
\text { für Ihre interne } \\
\text { Organisation? }\end{array}$ & \begin{tabular}{|l|} 
Die Abwicklung \\
vieler Vorgänge ist \\
aufwendig, weil mehr \\
Einzelvorgänge zu \\
bearbeiten sind und \\
seither nutzbare \\
Rationalisierungsef- \\
fekte wegfallen.
\end{tabular} & $\begin{array}{l}\text { Das bedeutet, dass die } \\
\text { früher routiniert- } \\
\text { eingespielte Stelle Leis- } \\
\text { tungsabrechnung/Zahl- } \\
\text { ungsverkehr nun die } \\
\text { unterschiedlichsten Rege- } \\
\text { lungen und Verfahrens- } \\
\text { weisen beachten muss. }\end{array}$ & $\begin{array}{l}\text { Statt mit zwei haben wir es } \\
\text { jetzt mit einer Vielzahl von } \\
\text { Personen zu tun, weil es bei } \\
\text { manchen Kostenträgern } \\
\text { mehrere Ansprechpartner } \\
\text { gibt, die oft erst kurze Erfahr- } \\
\text { ung auf dem Gebiet haben. } \\
\text { Das macht die Zusammenar- } \\
\text { beit aufwendiger. AuBerdem } \\
\text { werden zunehmend - wie } \\
\text { bei Jugendhilfe und Psychiat- } \\
\text { rie - durch die Kostenträger } \\
\text { Hilfeplangespräche ein- } \\
\text { berufen, was mit großem per- } \\
\text { sonellen Aufwand verbunden } \\
\text { ist. }\end{array}$ & $\begin{array}{l}\text { Für die interne Organisation } \\
\text { bedeutet das eine enorme } \\
\text { Steigerung der Komplexität, } \\
\text { die nur durch eine Dezentral- } \\
\text { isierung der Strukturen und } \\
\text { eine Bündelung und Stärkung } \\
\text { der regionalen Ebene zu } \\
\text { bewältigen ist. Für die Ver- } \\
\text { waltung steigt der Aufwand } \\
\text { insofern erheblich, als die } \\
\text { Kreise nach dem Ubergangs- } \\
\text { jahr } 2005 \text { nun zunehmend } \\
\text { eigene Wege zu gehen begin- } \\
\text { nen. }\end{array}$ & $\begin{array}{l}\text { Der administrative Mehrauf- } \\
\text { wand ist beträchtlich, insbe- } \\
\text { sondere für die Leistungs- } \\
\text { abrechnung, wegen der } \\
\text { veränderten Zuständigkeit für } \\
\text { die Qualitätsentwicklung und } \\
\text { wegen des erhöhten Aufwan- } \\
\text { des für die Planung. }\end{array}$ & \begin{tabular}{|l} 
Früher erfolgte ein Großteil \\
der Administration über die \\
LWVs. Jetzt müssen mit \\
jedem Landkreis \\
Verwaltungsaufgaben, Zahl- \\
ungsegelungen, Vereinbarun- \\
gen etc. ausgehandelt \\
werden. Es gibt Landkreise, \\
die zügig und kompetent ihre \\
neuen Aufgaben erledigen; \\
andere verursachen unnoti- \\
gen Verwaltungsaufwand \\
oder sind mit wenig kompe- \\
tenten Mitarbeitern besetzt.
\end{tabular} & $\begin{array}{l}\text { Die alltäglichen Verwal- } \\
\text { tungsabläufe funktionieren- } \\
\text { entgegen ersten Befürchtun- } \\
\text { gen -annähernd problemlos. } \\
\text { Das liegt sicher auch daran, } \\
\text { dass nun doch die allermeis- } \\
\text { ten Landkreise die seitherige } \\
\text { Abrechnungssoftware des } \\
\text { Landeswohlfahrtsverbandes } \\
\text { übernommen haben. }\end{array}$ \\
\hline $\begin{array}{l}\text { Ist das Ganze in } \\
\text { lhrem Hause mit } \\
\text { messbaren Kos- } \\
\text { tenerhöhungen } \\
\text { verbunden? }\end{array}$ & $\begin{array}{l}\text { Weil heute viele Ein- } \\
\text { zelvorgänge entste- } \\
\text { hen, kann man schon } \\
\text { von einem Mehrauf- } \\
\text { wand sprechen. }\end{array}$ & $\begin{array}{l}\text { Zur Frage der Messbarkeit } \\
\text { der Kostenerhöhung } \\
\text { würden wir meinen, dass } \\
\text { dies einen Personalmehr- } \\
\text { aufwand von ca. } 0,25 \text { VK- } \\
\text { Stelle ausmacht. Das sind } \\
\text { zusätzliche Personalko- } \\
\text { sten von etwa T } € 12 \text { im } \\
\text { Jahr. }\end{array}$ & $\begin{array}{l}\text { Es ist bisher nicht möglich, } \\
\text { den Mehraufwand finanziell } \\
\text { zu beziffern, weil immer } \\
\text { mehrere Stellen im Hause } \\
\text { betroffen sind (Wohngruppe, } \\
\text { Bewohnerverwaltung, Kasse } \\
\text { etc.). }\end{array}$ & $\begin{array}{l}\text { Man muss wohl davon aus- } \\
\text { gehen, dass dieser Prozess } \\
\text { auch mit Kostenerhöhungen } \\
\text { verbunden ist. Vermutlich ist } \\
\text { es noch zu früh, diese zu } \\
\text { bewerten. }\end{array}$ & \begin{tabular}{|l} 
Im Jahr 2005 konnte der \\
Mehraufwand bei der Leis- \\
tungsabrechnung nur mit 300 \\
Überstunden abgedeckt wer- \\
den. Der Mehraufwand für die \\
anderen Bereiche ist nicht \\
quantifizierbar.
\end{tabular} & \begin{tabular}{|l|} 
Die neue Zuständigkeit \\
bedeutet einen erheblichen \\
Mehraufwand für die Stiftung. \\
Nur durch den außerordentli- \\
chen Einsatz der Mitarbeiter \\
in der Verwaltung konnt \\
dieser Kraftakt ohne zusätzli- \\
che Personaleinstellungen \\
bewältigt werden.
\end{tabular} & Keine Angaben. \\
\hline $\begin{array}{l}\text { Erleben Sie, dass } \\
\text { jeder Landkreis } \\
\text { seine eigene Poli- } \\
\text { tik macht, oder ist } \\
\text { ein roter Faden } \\
\text { erkennbar? }\end{array}$ & $\begin{array}{l}\text { Wir haben keine } \\
\text { Probleme mit den } \\
\text { Landkreisen, mit } \\
\text { denen wir arbeiten. }\end{array}$ & $\begin{array}{l}\text { Es ist in der Tat so, dass } \\
\text { jeder Landkreis seine } \\
\text { eigenen Vorstellungen hat } \\
\text { und seine eigene Politik } \\
\text { betreibt. Ein roter Faden ist } \\
\text { in der Kostenreduzierung } \\
\text { erkennbar. Konkret: } \\
\text { Heimaufnahmen werden } \\
\text { verhindert oder verzögert. }\end{array}$ & $\begin{array}{l}\text { Wir erleben es tatsächlich so, } \\
\text { dass jeder Landkreis seine } \\
\text { eigene Politik macht. Die } \\
\text { Behindertenplanung sieht in } \\
\text { jedem Landkreis anders aus. } \\
\text { Die Beteiligung der Träger } \\
\text { und Selbsthilfe-/Ange- } \\
\text { hörigenorganisationen wird } \\
\text { ebentallls sehr unterschiedlich } \\
\text { gehandhabt. }\end{array}$ & $\begin{array}{l}\text { Wir erleben statt dem »roten } \\
\text { Faden« eher eine »neue } \\
\text { Unübersichtlichkeit«. Ein- } \\
\text { erseits gibt es eine zentrifu- } \\
\text { gale Tendenz, indem sich die } \\
\text { Kreise auf den Weg machen, } \\
\text { ihre Eigenständigkeit betonen } \\
\text { und erst einmal ihre eigenen } \\
\text { Positionen finden müssen. } \\
\text { Andererseits gibt es auch } \\
\text { eine zentripetale Tendenz zur } \\
\text { landesweiten interkommu- } \\
\text { nalen Abstimmung, was aller- } \\
\text { dings die örtlichen } \\
\text { Verhandlungen behindern } \\
\text { oder verzögern kann. }\end{array}$ & $\begin{array}{l}\text { Als roter Faden ist erkennbar, } \\
\text { die Kosten der Eingliede- } \\
\text { rungshilfe deutlich zu senken. } \\
\text { Ein positiver Effekt dieser } \\
\text { Reform ist, dass die Politik } \\
\text { vor Ort deutlich stärker in die } \\
\text { Verantwortung genommen } \\
\text { wird, die Belange für Men- } \\
\text { schen mit Behinderungen vor } \\
\text { Ort zu gestalten. Die Leis- } \\
\text { tungserbringer werden gege- } \\
\text { neinander ausgespielt. Die } \\
\text { Politik wollte Wettbewerb, } \\
\text { erhalten hat sie ein Preisdik- } \\
\text { tat der Kostenträger. vor Ort } \\
\text { zu gestalten. }\end{array}$ & $\begin{array}{l}\text { Jeder Landkreis beschäftigt } \\
\text { sich auf seine Weise mitt der } \\
\text { Behindertenhilfe und regelt } \\
\text { die Hilfeplanung individuell. } \\
\text { Die Eingliederungshilfe ist } \\
\text { jetzt nicht mehr ein »neutraler } \\
\text { Haushaltsposten« der Kom- } \\
\text { mune, sondern steht in } \\
\text { Konkurrenz zu anderen Leis- } \\
\text { tungen der Kommunen. Die } \\
\text { Landkreise versuchen, } \\
\text { Kosteneinsparungen zu } \\
\text { erzielen oder zumindest die } \\
\text { Kosten zu steuern. Als roter } \\
\text { Faden zeichnet sich ab: Die } \\
\text { Landkreise führen die Einzel- } \\
\text { fallsteuerung ein und treffen } \\
\text { Kostenübernahmen nach } \\
\text { sorgfältiger Prüfung der indi- } \\
\text { viduellen Sachlage. }\end{array}$ & \begin{tabular}{|l} 
In sozialplanerischer Hin- \\
sicht erleben wir nach einer \\
ersten Orientierungsphase, \\
dass die Landkreise ihre \\
Angebotsstruktur eigenstän- \\
dig gestalten wollen. Nach \\
unserem Kenntnisstand wer- \\
den derzeit durch den KVJS \\
in 11 Landkreisen Kreisbe- \\
hindertenpläne erarbeitet. \\
Ungelöst ist die Frage überre- \\
gionaler Planungserfordern- \\
isse, etwa in Ballungsge- \\
bieten oder dort, wo \\
Angebote kreisübergreifend \\
in Anspruch genommen wer- \\
den. Die Akteure auf der \\
öffentlichen Seite scheinen \\
noch immer mit der Klärung \\
der eigenen Rolle beschäftigt \\
zu sein.
\end{tabular} \\
\hline $\begin{array}{l}\text { Wie wird sich } \\
\text { diese politische } \\
\text { Entscheidung am } \\
\text { Ende für die } \\
\text { Betroffenen } \\
\text { auswirken? }\end{array}$ & Keine Angaben. & $\begin{array}{l}\text { Ob die Kommunalisierung } \\
\text { wirklich mehr Bürgernähe } \\
\text { für die Betroffenen bedeu- } \\
\text { tet, können wir selbst nicht } \\
\text { beurteilen. Wir haben den } \\
\text { Eindruck, dass die } \\
\text { behinderten Menschen } \\
\text { wieder mehr zu Bittstellern } \\
\text { werden und mit Antworten } \\
\text { wie: "Sie brauchen doch } \\
\text { kein Wohnheim! Sie kön- } \\
\text { nen doch im ambulant- } \\
\text { betreuten Wohnen leben « } \\
\text { abgefertigt werden. }\end{array}$ & Keine Angaben. & $\begin{array}{l}\text { Die Auswirkungen auf die } \\
\text { Betroffenen sind zwiespältig. } \\
\text { Die größere Nähe der Sozial- } \\
\text { verwaltung zur Lebenswelt } \\
\text { der behinderten Menschen } \\
\text { und die leichte Erreichbarkeit } \\
\text { sind positiv. Das Auseinan- } \\
\text { derdriften der Landkreise } \\
\text { hinsichtlich der Lebensbedin- } \\
\text { gungen für behinderte Men- } \\
\text { schen ist problematisch. }\end{array}$ & Keine Angaben. & Keine Angaben. & Keine Angaben. \\
\hline $\begin{array}{l}\text { Sonstige } \\
\text { Anmerkungen }\end{array}$ & $\begin{array}{l}\text { Es wäre wün- } \\
\text { schenswert, das } \\
\text { Abrechnungsver- } \\
\text { fahren zu verein- } \\
\text { heitlichen. }\end{array}$ & Keine Angaben. & Keine Angaben. & $\begin{array}{l}\text { Es würde vieles verein- } \\
\text { fachen, wenn im Landesaus- } \\
\text { führungsgesetz zum SGB XII } \\
\text { eine Pflicht der Kreise zur } \\
\text { gemeinsamen Regelung und } \\
\text { Abstimmung auf wichtigen } \\
\text { Feldern festgelegt würde. }\end{array}$ & \begin{tabular}{|l} 
Für die kurzfristige Entlastung \\
der öffentlichen Kassen wur- \\
den bewährte Strukturen zer- \\
schlagen, ohne dass neue \\
und tragfähige schon \\
bestünden und/oder erkenn- \\
bar sind. Völlig unklar ist die \\
Bearbeitungsstruktur von \\
Fragestellungen mit überre- \\
gionalem Charakter.
\end{tabular} & Keine Angaben. & Keine Angaben. \\
\hline
\end{tabular}




\title{
Kommunalisierung der Eingliederungshilfe in Baden-Württemberg: was die Leistungsträger sagen
}

\begin{tabular}{|c|c|c|c|}
\hline & $\begin{array}{l}\text { Ulrike Dimmler-Trumpp, Sozialdezernentin, } \\
\text { Landkreis Tübingen }\end{array}$ & $\begin{array}{l}\text { Diana E. Rädler, Sozialdezernentin, } \\
\text { Landkreis Ravensburg }\end{array}$ & $\begin{array}{l}\text { Andreas Bauer, Sozialdezernent, Landkreis } \\
\text { Reutlingen }\end{array}$ \\
\hline $\begin{array}{l}\text { Ist der Übergang der } \\
\text { Aufgaben von der } \\
\text { Landes-auf die Land- } \\
\text { kreisebene aus Ihrer } \\
\text { Sicht abgeschlossen? }\end{array}$ & $\begin{array}{l}\text { Der Aufgabenübergang ist aus unserer Sicht } \\
\text { abgeschlossen. }\end{array}$ & $\begin{array}{l}\text { Insgesamt ist der Aufgabenübergang } \\
\text { noch nicht abgeschlossen. Es ist } \\
\text { vielmehr eine Weiterentwicklung der } \\
\text { Behindertenhilfe durch die Kommu- } \\
\text { nen erforderlich. }\end{array}$ & $\begin{array}{l}\text { Der Aufgabenübergang war sehr schnell } \\
\text { abgeschlossen. Probleme gab es im ersten } \\
\text { halben Jahr vor allem bei administrativen } \\
\text { Aufgaben wie der korrekten und zügigen } \\
\text { Begleichung der Rechnungen oder der Ver- } \\
\text { buchung der Rechnungen mit dem vorhan- } \\
\text { denen EDV-System. Das sind aber Begleiter- } \\
\text { scheinungen, die zu erwarten waren. }\end{array}$ \\
\hline $\begin{array}{l}\text { Was wollen Sie als } \\
\text { Träger der } \\
\text { Eingliederungshilfe } \\
\text { für Ihren Landkreis } \\
\text { erreichen? }\end{array}$ & $\begin{array}{l}\text { Wir sind dabei, die wohnortnahe Versorgung } \\
\text { deutlich auszubauen. Ziel soll es sein, dass } \\
\text { zukünftig allen behinderten Menschen aus dem } \\
\text { Landkreis ein Angebot zum Wohnen bzw. zur } \\
\text { Tagesstrukturierung im Landkreis gemacht wer- } \\
\text { den kann. Lediglich bei ganz speziellen Ange- } \\
\text { boten wird es hier auch weiterhin eine } \\
\text { landkreisübergreifende Zusammenarbeit mit } \\
\text { den Einrichtungen geben. }\end{array}$ & $\begin{array}{l}\text { Durch Zusammenführung der Sach- } \\
\text { gebiete Eingliederungshilfe des Kreis- } \\
\text { sozialamtes und Schwerbehinderten- } \\
\text { recht des Versorgungsamtes soll im } \\
\text { Sozialdezernat ein Amt für Menschen } \\
\text { mit Behinderung gebildet werden, um } \\
\text { für Menschen mit Behinderung Hilfen } \\
\text { aus einer Hand und unter einem Dach } \\
\text { zu gewähren. Selbstverständlich ist es } \\
\text { eine weitere Aufgabe, trotz steigender } \\
\text { Fallzahlen die Kosten im Griff zu } \\
\text { halten. Bezüglich der in diesem } \\
\text { Zusammenhang häufig diskutierten } \\
\text { Standards ist vor allem auch der } \\
\text { Bundesgesetzgeber gefragt. }\end{array}$ & $\begin{array}{l}\text { Die Eingliederungshilfe ist ein großer } \\
\text { Schwerpunkt unserer Arbeit in den nächsten } \\
\text { Jahren. Wir wollen für die behinderten Men- } \\
\text { schen ein passendes, differenziertes Hil- } \\
\text { feangebot sicherstellen. Dazu gehört auch } \\
\text { die Beratung und Hilfeplanung im Einzelfall. } \\
\text { Wir sehen uns als ersten Ansprechpartner } \\
\text { für die behinderten Menschen. Wichtig ist } \\
\text { auch der Umbau der Versorgungsstrukturen. } \\
\text { Von einem gemeindenahen ambulanten und } \\
\text { stationären Hilfesystem wie in der Altenhilfe } \\
\text { ist die Behindertenhilfe noch weit entfernt. } \\
\text { Bei unseren großen Trägern sehe ich eine } \\
\text { große Aufgeschlossenheit zum Umbau und } \\
\text { zur Dezentralisierung, auch in andere } \\
\text { Herkunftskreise. Das wird aber ein längerer } \\
\text { Prozess sein. }\end{array}$ \\
\hline $\begin{array}{l}\text { Was läuft aus Ihrer } \\
\text { Sicht besser, was } \\
\text { schlechter als mit der } \\
\text { vorherigen Rege- } \\
\text { lung? }\end{array}$ & $\begin{array}{l}\text { Die Nähe zum behinderten Mitbürger ist ein } \\
\text { großer Vorteil für beide Seiten und ermöglicht } \\
\text { über die Hilfeplanung ein viel passgenaueres } \\
\text { Angebot. Die Menschen kommen häufig zu } \\
\text { »ihrem« Sachbearbeiter. Man kennt sich per- } \\
\text { sönlich. Auch der enge Kontakt zu den Leis- } \\
\text { tungserbringern vor Ort schafft ein angenehmes } \\
\text { und vertrauensvolles Klima. Durch die Finanz- } \\
\text { und Planungsverantwortung des Landkreises } \\
\text { können die örtlichen Gegebenheiten besser } \\
\text { berücksichtigt und somit auch ein Umbau der } \\
\text { Versorgungsstrukturen gezielter angegangen } \\
\text { werden. }\end{array}$ & $\begin{array}{l}\text { Positiv an der neuen Regelung ist, } \\
\text { dass der Landkreis bei der Planung } \\
\text { nun näher am Geschehen dran ist. } \\
\text { Wenn man vor Ort ist, beurteilt man } \\
\text { viele Dinge anders und richtiger. } \\
\text { Dadurch, dass viele Träger in } \\
\text { unserem Landkreis überregional } \\
\text { arbeiten, ist es für den Landkreis } \\
\text { Ravensburg schwierig, einen Über- } \\
\text { blick über das Betätigungsfeld jedes } \\
\text { Trägers zu bekommen. }\end{array}$ & $\begin{array}{l}\text { Insgesamt bewerte ich den Aufgabenüber- } \\
\text { gang positiv. Erst durch die Nähe zu den Ein- } \\
\text { richtungen vor Ort und zu den } \\
\text { Hilfesuchenden lassen sich in größerem } \\
\text { Umfang individuell die passenden Lösungen } \\
\text { entwickeln. Die Möglichkeit, in begrenztem } \\
\text { Umfang örtliche Regelungen zutreffen, sehe } \\
\text { ich in erster Linie als Chance und nicht, wie } \\
\text { vereinzelt zu hören ist, als »Erosion« bisheri- } \\
\text { ger Standards. }\end{array}$ \\
\hline
\end{tabular}

\section{Soziale Arbeit in Bewegung}

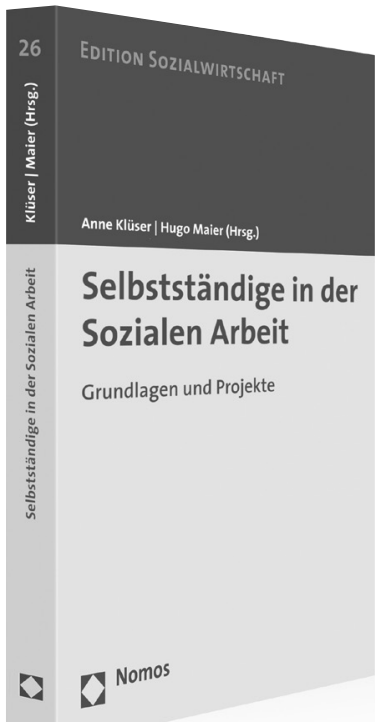

\author{
Selbstständige in der Sozialen Arbeit \\ Grundlagen und Projekte \\ Herausgegeben von Dr. Anne Klüser und Prof. Dr. Hugo Maier \\ 2009, 263 S., brosch., 44, - €, ISBN 978-3-8329-4111-6 \\ (Edition Sozialwirtschaft, Bd. 26)
}

Berufliche Selbstständigkeit in der Sozialen Arbeit: Heute ist kaum noch ein Bereich denkbar, in dem dies nicht möglich wäre. In vielen Feldern Sozialer Arbeit sind Selbstständige inzwischen etabliert und zu einer festen Größe avanciert. Sie setzen neue Standards, fordern die traditionellen Wohlfahrtsverbände heraus und bringen so Bewegung in die Soziale Arbeit.

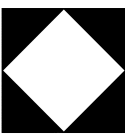

